\title{
Orbital stability of planets in binary systems: A new look at old results
}

\author{
J. Eberle, M. Cuntz and Z. E. Musielak \\ Department of Physics, University of Texas at Arlington, \\ Arlington, TX 76019-0059, USA \\ email: cuntz@uta.edu, zmusielak@uta.edu
}

\begin{abstract}
About half of all known stellar systems with Sun-like stars consist of two or more stars, significantly affecting the orbital stability of any planet in these systems. This observational evidence has prompted a large array of theoretical research, including the derivation of mathematically stringent criteria for the orbital stability of planets in stellar binary systems, valid for the "coplanar circular restricted three-body problem". In the following, we use these criteria to explore the validity of results from previous theoretical studies.
\end{abstract}

Keywords. Astrobiology, methods: numerical, binaries, planetary systems

\section{Introduction}

Observational evidence for the existence of planets in stellar binary (and higher order) systems has been given by Patience et al. (2002), Eggenberger et al. (2004, 2007), and others. Eggenberger et al., presented data for more than thirty systems, mostly wide binaries, as well as several triple star systems, with separation distances as close as $20 \mathrm{AU}$ (GJ 86). These findings are consistent with previous theoretical results which showed that planets can successfully form in binary (and possibly multiple) stellar systems (e.g., Kley 2001, Quintana et al. 2002), known to occur in high frequency in the local Galactic neighborhood (Duquennoy \& Mayor 1991, Lada 2006, Raghavan et al. 2006). More recently, Bonavita \& Desidera (2007) performed a statistical analysis for binaries and multiple systems concerning the frequency of hosting planets, leading to the conclusion that there is no significant statistical difference between binary systems and single stars. The fact that planets in binary systems are now considered to be relatively common is also implied by the recent detection of debris disks in various main-sequence stellar binary systems using the Spitzer Space Telescope (Trilling et al. 2007). The research team observed 69 main-sequence binary star systems in the spectral range of A3 to F8.

In our previous work, we studied the stability of both S-type and P-type orbits in stellar binary systems, and deduced orbital stability limits for planets (Musielak et al. 2005). P-type orbits lie well outside the binary system, where the planet essentially orbits the center of mass of both stars, whereas S-type orbits lie near one of the stars, with the second star acting as a perturbator. The limits of stability were found to depend on the mass ratio between the stellar components. This topic has recently been revisited by Cuntz et al. (2007) and Eberle et al. (2008a, 2008b), who used the concept of Jacobi's integral and Jacobi's constant (Szebehely 1967, Roy 2005) to deduce stringent criteria for the stability of planetary orbits in binary systems for the special case of the "coplanar circular restricted three-body problem". These criteria are used to contest previous results on planetary orbital stability in binary systems available in the literature. 


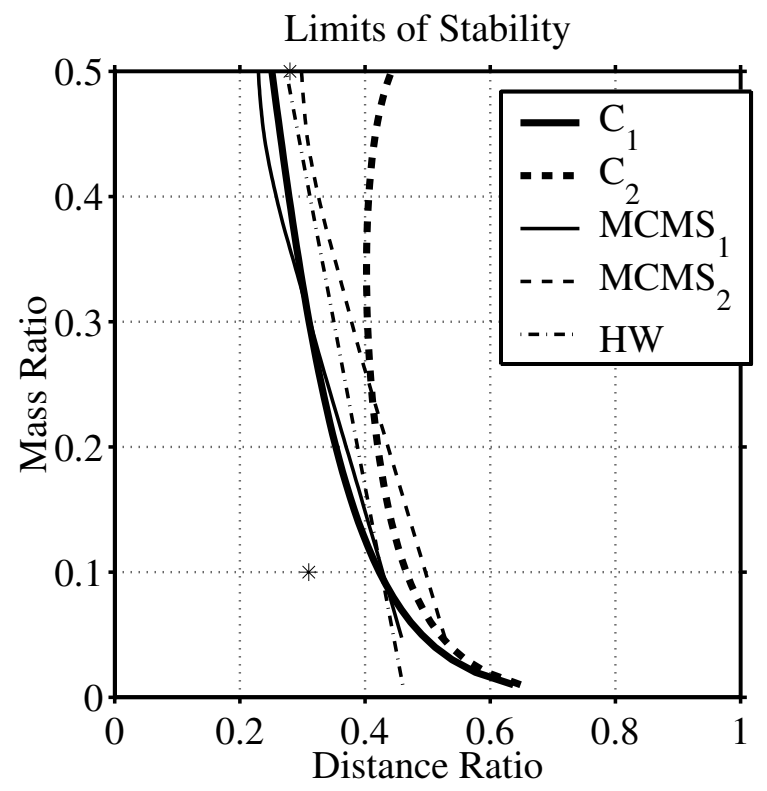

Figure 1. Limits of stability for planetary orbits for different mass ratios $\mu$ between the secondary star and the sum of the two stellar masses. We show the result based on Jacobi's constant $C_{1}$ (thick solid line) (criterion of stability) and based on Jacobi's constant $C_{2}$ (thick dashed line). For comparison, we also show the dividing lines between the regions of stability and marginal stability (thin solid line) $\left[\mathrm{MCMS}_{1}\right]$ and between marginal stability and instability $\left[\mathrm{MCMS}_{2}\right]$ (thin dashed line) previously obtained by Musielak et al. (2005). The two asterices (*) indicate the stability limit by David et al. (2003) corresponding to $10^{6}$ orbits. The stability limit from the earlier work by Holman \& Wiegert (1999) [HW] is depicted as a thin dash-dotted line.

\section{Results and Conclusions}

By focusing on the coplanar circular restricted three-body problem, we have been able to deduce absolute stability limits for small-mass planets, which only depend on the mass ratio between the two stellar components (see Fig. 1). The analysis by Eberle et al. (2008a) elucidates the physical backround of these limits. They are closely related to the so-called "surfaces of zero velocity" (or zero velocity contours) that form the boundaries of regions where the planet must be found. Thus, planets are unable to escape from these regions, preventing them from leaving the system entirely or from being captured by the other stellar component. This property is tantamount to orbital stability, although it does not necessarily imply stability in the sense of quasi-periodicity. The rationale of the contour is that it limits the allowable region of the planet due to its limited available energy.

In the following, we compare the absolute stability limit of Cuntz et al. (2007) and Eberle et al. (2008b) to results from time-dependent orbital stability simulations obtained by Holman \& Wiegert (1999), David et al. (2003), and Musielak et al. (2005) (see Fig. 1). 
The comparison of this newly found absolute stability limit with the stability limits previously deduced by Musielak et al. shows that their numerically derived stability limit largely agrees with the $C_{1}$-related stability criterion (Cuntz et al. 2007, Eberle et al. 2008 b), although for a small range of mass ratios their stability criterion is too strict. In addition, there are discrepancies between our $C_{2}$ criterion for unstable orbits and their criterion for marginally stable / unstable orbits. Previous work by Holman \& Wiegert (1999) considered a large range of eccentricities for the stellar binary components. For circular orbits, their stability limit is similar to the $C_{1}$-based stability limit in our study as well as the stability limit of Musielak et al. (2005), although a careful analysis shows that small, but noticeable differences exist as the Holman \& Wiegert criterion is somewhat less strict for mass ratios between $\mu=0.5$ and 0.1 , but too strict for mass ratios of less than 0.1 - at least as viewed based on the polynomial fit given by the authors.

Other recent results have been given by David et al. (2003). They investigated the range of orbital stability for a similar parameter range as previously discussed. Here the orbital stability of an Earth-mass planet around a solar-mass star is studied in the presence of a companion star for a range of companion masses between 0.001 and $0.5 M_{\odot}$. David et al. (2003) consider both circular and elliptical orbits, and derive expressions for the expected ejection time of the planet from the system. For fixed companion masses, the ejection time is found to be a steep function of the periastron distance (or orbital radius for circular orbits) to the primary star. David et al. predict that the domain of orbital stability gets progressively smaller over time according to the logarithm of the time of simulation, even though some cases in their study do not seem to indicate this type of behavior. Note that their principle finding of a progressively decreasing stability limit is fundamentally inconsistent with the result from the Cuntz et al. (2007) and Eberle et al. (2008b) studies that yield an analytically derived absolute limit of stability, although those cases solely focus on the restricted three-body problem. For $\mu=0.3$ and zero eccentricity, David et al. (2003) deduce a stability limit of $\rho_{0}=0.31 \pm 0.04$ for $10^{6}$ orbits, which is well inside the analytically defined stability region obtained in our study.

In conclusion, it is found that the comparison of the results by Cuntz et al. (2007) and Eberle et al. (2008b) to those previously obtained by Holman \& Wiegert (1999), David et al. (2003), and Musielak et al. (2005), show that their criteria for orbital stability are in good agreement with our analytical criterion, although some small, but noticeable differences exist. For planets which do not fulfill our stringent criterion of orbital stability, stability may still be possible for a significant period of time. However, in this case the outcome must carefully be studied by employing long-term simulations and may depend on various factors, such as, e.g., the mass and orbital starting position of the planet.

\section{References}

Bonavita, M. \& Desidera, S. 2007, A\& $A, 468,721$

Cuntz, M., Eberle, J., \& Musielak, Z.E. 2007, ApJ (Letters), 669, L105

David, E. -M., Quintana, E. V., Fatuzzo, M., \& Adams, F. C. 2003, PASP, 115, 825

Duquennoy, A., \& Mayor, M. 1991, A\&A, 248, 485

Eberle, J., Cuntz, M., \& Musielak, Z. E. 2008a, in: K. Meech et al. (eds.), Bioastronomy 2007: Molecules, Microbes and Extraterrestrial Life (San Francisco: ASP), in press

Eberle, J., Cuntz, M., \& Musielak, Z. E. 2008b, A\& A, submitted

Eggenberger, A., \& Udry, S. 2007, in: N. Haghighipour (ed.), Planets in Binary Star Systems (New York: Springer), in press

Eggenberger, A., Udry, S., \& Mayor, M. 2004, A\& A, 417, 353

Holman, M. J., \& Wiegert, P. A. 1999, AJ, 117, 621 
Kley, W. 2001, in: H. Zinnecker \& R.D. Mathieu (eds.), The Formation of Binary Stars, IAU Symp. 200 (San Francisco: ASP), p. 511

Lada, C. J. 2006, ApJ (Letters), 640, L63

Musielak, Z. E., Cuntz, M., Marshall, E. A., \& Stuit, T. D. 2005, A\&A, 434, 355

Patience, J., et al. 2002, ApJ, 581, 654

Quintana, E. V., Lissauer, J. J., Chambers, J. E., \& Duncan, M. J. 2002, ApJ, 576, 982

Raghavan, D., et al. 2006, ApJ, 646, 523

Roy, A. E. 2005, Orbital Motion (Bristol and Philadelphia: Institute of Physics Publ.)

Szebehely, V. 1967, Theory of Orbits (New York and London: Academic Press)

Trilling, D. E., et al. 2007, ApJ, 658, 1289 